Rapid Reviews COVID-19•

\title{
Review 1: "Protection \\ Across Age Groups of \\ BNT162b2 Vaccine Booster \\ against Covid-19 "
}

\section{Arthur Reingold ${ }^{1}$}

${ }^{1}$ UC Berkeley School of Public Health

Published on: Nov 06, 2021

License: Creative Commons Attribution 4.0 International License (CC-BY 4.0). 


\section{$\underline{\text { RR:C19 Evidence Scale rating by reviewer: }}$}

- Strong. The main study claims are very well-justified by the data and analytic methods used. There is little room for doubt that the study produced has very similar results and conclusions as compared with the hypothetical ideal study. The study's main claims should be considered conclusive and actionable without reservation.

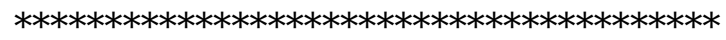

\section{Review:}

This study by investigators in Israel assessed the effect of booster doses of the BNT162b2 COVID-19 vaccine on the rate of COVID-19 illnesses and deaths in a large observational cohort study. The results provide compelling evidence supporting the effectiveness of booster doses in individuals across the age spectrum, beginning $12+$ days following the receipt of the booster dose. While the authors did not calculate the effectiveness of booster doses, the reductions in the rate of important COVID-19 outcomes are consistent with a very high level of vaccine booster dose effectiveness. Unfortunately, the study was unable to provide estimates of the impact of booster doses among finer gradients of age above 60 years, presumably because of small numbers of individuals. Additionally, the manuscript could not provide estimates of the impact of booster doses on individuals with immuno-compromising or other underlying illnesses, presumably because this information was unavailable to the investigators. Although it is difficult to exclude the effect of confounding and various forms of selection and information bias on the results entirely, the study provides strong evidence that booster doses of the BNT162b2 vaccine have their intended effect. 